\title{
Effect of local context of responding on human judgment of causality
}

\author{
PHIL REED \\ University of Sussex, Brighton, England
}

\begin{abstract}
Two experiments examined the effect of various relationships between a response (pressing the space bar of a computer) and an outcome (a triangle flashing on a screen) on judgments of the causal effectiveness of the response. In Experiment 1, when responses were required to be temporarily isolated from each other prior to an outcome, ratings of the causal effectiveness of the responses were higher than in a condition in which the probability of an outcome following a response was the same but in which no temporal isolation was required. In Experiment 2, when a number of responses were required to be emitted temporally close to the outcome, ratings of the causal effectiveness of the responses were lower than in a condition in which the probability of an outcome following a response was the same but in which no temporal proximity was required. These results suggest that, in addition to the overall probability that an outcome will follow a response, the local context of responding at the time an outcome is presented is critical in influencing ratings of causal effectiveness.
\end{abstract}

Traditional views of human causality judgment have focused on the likelihood that humans perform a comparison of the probability of the outcome, given the presence or absence of the predictor (in this case, a response). If the probability of an outcome, given the occurrence of a cue (e.g., a response), is greater than the probability that the outcome will be absent when a cue occurs, then a positive assessment of the contingent relationship between cue and outcome will be given. If the converse relationship holds, then the judgment regarding the causal efficacy of the cue will (where negative judgments are permitted) be negative (Shaklee \& Tucker, 1980; Ward \& Jenkins, 1965; see Neunaber \& Wasserman, 1986, for further discussion).

Recently, it has been established that many of the factors that influence the course of conditioning also influence human judgments of causal efficacy (Alloy \& Abramson, 1979; Chapman \& Robbins, 1990; Shanks \& Dickinson, 1987; Wasserman, 1990). Evidence for this functional equivalence has been provided from a variety of sources, most notably in relation to the influence that the contiguity and the contingency between response and outcome have on the judgment of the causal effectiveness of that response (e.g., Shanks, Pearson, \& Dickinson, 1989; Wasserman, Chatlosh, \& Neunaber, 1983).

For the present purposes, the traditional view differs in no important detail from that derived from views of associative learning (e.g., Shanks \& Dickinson, 1987; Wasserman, 1990). It should be noted that, in the former view, the values of the probability of an outcome, given

I would like to thank David Shanks and two anonymous reviewers for their comments on an earlier version of this paper. Requests for reprints should be addressed to P. Reed, Department of Psychology, University College London, Gower Street, London WC1 6BT, U.K. the occurrence of a cue, and the probability of an outcome in the absence of a cue are computed independently of each other, and hence, such a view cannot account for studies that show that cues may interact to determine the level of perceived causal effectiveness in a given situation (see Chapman \& Robbins, 1990; Shanks, 1985).

The influence on conditioning of the contingency that relates a response to an outcome (reward) has been extensively studied in terms of schedules of reinforcement. This area provides a highly documented, and relatively well understood, background (for reviews, see Ferster \& Skinner, 1957; Zeiler, 1977) from which to extend the investigation into the effect of various relationships between a response and the outcome on human judgments of causality. Dickinson (1985) has suggested that the schedule of reinforcement influences nonhuman perception of the causal efficacy of a response. If a functional equivalence between human causal attribution and nonhuman conditioning is accepted, and if it is further accepted that the contingency that relates a response to an outcome is critical in human causal attribution, then the schedule that relates the response to an outcome might also be important in influencing such causal attributions.

Reed (1992) has conducted an initial investigation of the influence of different schedules on the judgment of causal effectiveness of a response. This report focused on the effect of two types of schedules: a variable-interval (VI) schedule, in which the first response emitted after a certain temporal interval had elapsed produced an outcome (responses prior to the interval criteria had no effect) and the temporal interval varied from outcome to outcome around a mean, and a variable-ratio (VR) schedule, in which a specified number of responses had to be made before an outcome was presented; the number of responses varied from outcome to outcome around a mean. This study indicated that when the response was 
related to the outcome according to a VI schedule, judgments of the causal effectiveness of responses performed on that schedule were high, relative to the level of causal effectiveness attributed to a response emitted during exposure to a VR schedule that was yoked to the VI schedule in terms of responses required per outcome. Present accounts of human causality judgments (e.g., Shanks, 1985; Shanks \& Dickinson, 1987; Ward \& Jenkins, 1965; Wasserman, 1990) cannot accommodate this result easily. The problem faced by both "traditional" and "associative" views, in light of the results obtained by Reed (1992), is that, in conditions in which the number of responses required to produce an outcome are identical, different judgments of the causal effectiveness of a response were noted when the rules relating a response to an outcome differed with respect to whether they were determined by a VI or a VR schedule.

A possible explanation for these results stems from research carried out to determine why VR and VI schedules maintain different response rates in nonhumans (see, e.g., Peele, Casey, \& Silberberg, 1984). It might be assumed that subjects perform successive responses with a variety of interresponse intervals. Due to the temporal nature of a VI schedule, an outcome is increasingly likely to follow a response as the interresponse interval increases (see Morse, 1966). This means that on a VI schedule relating responses to outcomes, the longer the interresponse interval, the more likely it will be that an outcome will follow the response. Given this, an interval-based rule may tend to produce relatively large numbers of outcomes if responses are made in temporal isolation from other responses. In contrast, the VR rule does not selectively tend to produce outcomes after temporally separated responses; thus, on a VR schedule, it is equally probable that a subject may have been responding a lot or a little, prior to the outcome.

If a response that occurs in temporal isolation from other responses produces an outcome, this response may be perceived to be more causally effective, because the subject may make the judgment that one response is sufficient to produce an outcome when that response was temporally isolated, but may take into account a number of responses if they are performed together immediately prior to an outcome. If a number of responses are produced in close temporal proximity to the outcome, the subject might assume that more than one response is necessary to produce the outcome and, consequently, lower the rating of causal effectiveness of individual responses. Thus, the probability of an outcome, given the occurrence of a response, may be judged over a limited period of time prior to the outcome, or temporally isolated responses may be given a relatively greater weight in any calculation of probabilities than may responses performed in the context of many other responses.

The present study represents an attempt to examine the "local context" view of causal attribution directly. This was done by studying the influence that the rates of responses produced prior to an outcome on the ratings of causal effectiveness. If response rate is very low prior to the outcome, then ratings of the causal efficacy of the response should be high relative to a condition in which there is the same probability of an outcome following a response, but in which response rates are not low prior to the delivery of an outcome. In contrast, should response rates be very high prior to an outcome, then the rating of the causal effectiveness of a response would be low relative to a condition matched in terms of the probability of an outcome following a response, but in which response rate was not very high prior to an outcome.

\section{EXPERIMENT 1}

In studies of instrumental learning on free-operant schedules of reinforcement, it is possible to arrange a contingency between responding and reinforcement such that reinforcement is only delivered for a response if that response is separated from the preceding response by a certain minimum time. This contingency is referred to as a differential reinforcement of low rate (DRL) schedule (see Ferster \& Skinner, 1957). Such a schedule can be adapted for use in the study of human judgment of causal effectiveness. If the local context view of causal attribution is an accurate account of the processes of causal attribution, responses produced on a DRL-like contingency should be rated as more causally effective than those produced on a schedule matched in terms of the probability that a response be followed by an outcome, but lacking the temporal separation requirement (i.e., a VR schedule yoked to the DRL contingency in terms of responses per outcome). This hypothesis was tested in the first experiment. Ratings of the causal effectiveness of responses produced on a VI schedule yoked in terms of the temporal distribution of outcomes to the DRL schedule were examined, so that the effect of spacing of outcomes on judgments of causal effectiveness could be investigated independently of the effect of response rate.

\section{Method}

Subjects. Sixteen subjects ( 12 female and 4 male) were recruited on and around the Sussex University campus. All subjects were volunteers, and none was paid for participating. The subjects had an age range of 18-35.

Apparatus. The experiment was conducted in an unfurnished room. The subject sat at a table, on which was placed a BBC computer that controlled a video display screen $24 \mathrm{~cm}$ wide $\times 17 \mathrm{~cm}$ high. This was placed approximately $50 \mathrm{~cm}$ in front of the subject. The subjects could use a computer keyboard (also in front of them) to respond to the instructions given on the video screen.

Procedure. At the start of the experiment, all the subjects were given the following instructions (modified from Shanks et al., 1989) on the video screen (responses that the subjects were required to make are presented in parentheses).

Please read the following instructions very carefully. Take as much time as you like. Your task in this experiment is to judge the extent to which you can cause something to happen on the computer screen.

There will be a triangle on the screen like this.

The outline of a triangle with sides $5 \mathrm{~cm}$ long was presented on the screen. The next instruction was as follows: 
"Now press the SPACE BAR and see what happens."

When the subject pressed the space bar, the whole triangle lit up for $100 \mathrm{msec}$; this constituted the outcome. After the outcome had occurred, the outline of the triangle remained on the screen as before. Then the instructions continued:

"And now press it a few more times."

The subject could press the space bar and observe the triangle flashing four more times. Then the instructions continued.

Your task in this experiment is to find out whether pressing the SPACE BAR has any effect on whether or not the triangle lights up. At any time you may choose whether or not to press the SPACE BAR. You can press it as often or as little as you like. However, because of the nature of the task it is to your advantage to press it some of the time and not to press it some of the time.

Sometimes the triangle will flash when you press the SPACE BAR, and sometimes it will not. You must judge the extent to which pressing the SPACE BAR is the cause of the triangle lighting up.

You will be given a number of different problems, each lasting for two or three minutes. The relationship between pressing the SPACE BAR and whether or not the triangle lights up will be constant within each problem, but may well differ from one problem to the next

At the end of each problem you will be asked to given an estimate on a rating scale of the extent to which you think that pressing the SPACE BAR caused the triangle to light up during that problem.

The first problem commenced after these instructions. Each subject was exposed to all three conditions in the present experiment. The DRL schedule was always presented first. In this condition, an outcome (a flash of the triangle) was presented immediately after a response on the space bar, provided that at least $5 \mathrm{sec}$ had elapsed since the last response or triangle flash. A response before $5 \mathrm{sec}$ had elapsed would reset the DRL time requirement. The number of responses made before each successive outcome in the DRL schedule was recorded and these numbers were played back to the subject as the successive ratio requirements in the VR schedule. Thus, the subjects had to make the same number of responses per outcome in the VR as in the DRL condition. The successive intervals between outcomes in the DRL condition were recorded, and these became the successive interval requirements for the VI condition. In this condition, the first response made after the interval elapsed produced the triangle flash; thus, the temporal intervals between outcomes were approximately equal in the DRL and VI conditions. For half the subjects, the VR schedule was presented second and the VI schedule was presented last. For the other half of the subjects, the order of presentation of the VR and VI conditions was reversed. After each condition had concluded, the subjects were asked to make a judgment of the causal efficacy of the responses made in that condition. The following instructions were presented:

Type in a number to indicate your judgment of the extent to which pressing the SPACE BAR caused the triangle to light up. Use a scale from 0 to 100 . Zero indicates that the SPACE BAR had no effect on whether the triangle lit up, and 100 means that the SPACE BAR always caused the triangle to light up. Press the RETURN key after typing in your judgement.

Your Judgment?

After the subject had typed a number, the next condition was presented. The DRL condition lasted for $2 \mathrm{~min}$, and the VR and VI conditions lasted until either the same number of responses and outcomes or outcomes, respectively, as in the DRL condition had been made and obtained.

\section{Results and Discussion}

Table 1 displays the number of responses per minute, the number of outcomes obtained per minute, and the probability that a response would be followed by an outcome, for the three conditions in Experiment 1. The subjects responded faster in the VI condition than in the VR
Table 1

Mean Responses per Minute, Outcomes per Minute, and Probability That an Outcome Would Follow a Response $(\mathbf{O} \mid \mathbf{R})$, for all Conditions in Experiment 1

\begin{tabular}{lrrr}
\hline & DRL & VR & \multicolumn{1}{c}{ VI } \\
\hline Responses/minute & 33.88 & 43.71 & 48.97 \\
Outcomes/minute & 3.88 & 4.84 & 3.88 \\
Probability $(O \mid R)$ & 0.19 & 0.19 & 0.11 \\
\hline
\end{tabular}

Note-DRL $=$ differential reinforcement of low rate; $V R=$ variable ratio; $\mathrm{VI}=$ variable interval

condition and, in turn, responded faster in the VR condition than in the DRL condition. However, an analysis of variance (ANOVA) conducted on these data revealed no statistically significant difference between the conditions $(p>.10)$. A greater number of outcomes were obtained per minute in the VR condition than in either the VI or the DRL condition; the latter two conditions had the same number of outcomes per minute. These scores were analyzed with an ANOVA, which revealed a significant difference between the conditions $[F(2,30)=961.00$, $p<.001]$. Examination of the probability that an outcome would follow a response reveals that this was less likely in the VI than in the VR and DRL conditions (this probability being the same in the latter two conditions). An ANOVA of these data revealed a significant difference between the conditions $[F(2,30)=6.60, p<.01]$.

Figure 1 displays the mean rating of the causal effectiveness of a response in each condition. Inspection of these data demonstrates that the responses in the DRL condition were rated more causally effective in the VR and VI conditions. Ratings of the causal effectiveness of the response were similar in the latter two conditions. These results were analyzed with an ANOVA, which revealed that the difference between the contingencies approached conventional levels of significance $[F(2,30)=3.18$, $.08>p>.07$ ]. Although this difference (narrowly) failed to reach conventional levels of significance, it was in the predicted direction. Inspection of the individual data reveals that 14 of the 16 subjects rated responses in the DRL condition as at least as causally effective as responses in either of the other two conditions.

Given this, it seemed appropriate to analyze these data further. The ratings of the causal effectiveness of responses in the conditions were subsequently analyzed by means of Tukey's honestly significant difference (HSD) tests, in which the pooled error term from the analysis above was used $\left(M S_{e}=300.46\right)$; this revealed that the responses in the DRL condition were rated as being significantly more effective than those in both the VR $(p<.05)$ and the VI $(p<.05)$ conditions. The ratings of causal effectiveness did not differ in the VR and VI conditions $(p>.05)$

These results are consistent with the prediction, made on the basis of the local context view, that responses in the DRL condition would be rated more causally effective than those in the VR condition, despite equal probabilities that an outcome would follow a response in the 


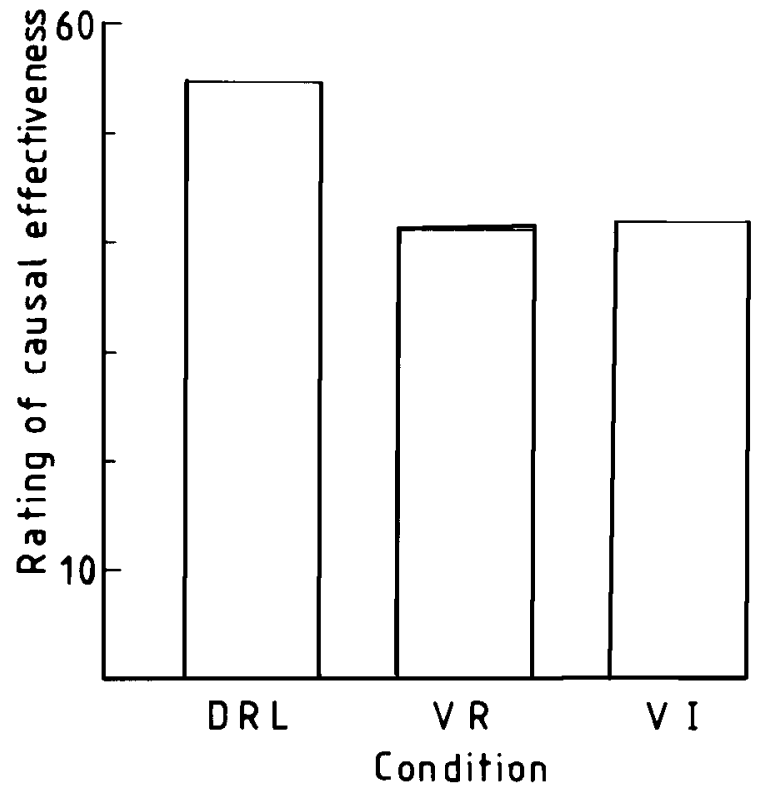

Figure 1. Results from Experiment 1: Mean ratings of the causal effectiveness of responses performed in each condition. DRL, differential reinforcement of low rate; VR, variable ratio; VI, variable interval.

two conditions. The relatively well-spaced temporal distribution of outcomes in the DRL condition compared to the VR condition was probably not the sole determinant of the higher rating given in the DRL condition, since ratings of causal effectiveness of responses in the DRL condition were higher than ratings of responses produced during exposure to a VI condition that was yoked with respect to the temporal distribution of outcomes.

\section{EXPERIMENT 2}

In addition to the arrangement of a schedule that requires temporally isolated responses, it is possible to arrange a schedule that requires responses to be temporally close. Such a schedule is referred to as a differential reinforcement of high rate (DRH) schedule. This contingency only arranges an outcome if a number of responses have been produced in close temporal proximity (see Ferster \& Skinner, 1957; Reed, 1989). On such a schedule, the local context view of causal attribution suggests that responses will be rated as less causally effective than those emitted on a schedule matched in terms of the probability of an outcome following a response, but with no requirement that groups of responses occur in close temporal proximity to the outcome. This suggestion was tested in the second experiment.

\section{Method}

Subjects and Apparatus. Sixteen subjects participated in this experiment ( 8 males and 8 females). Their ages ranged from 18 to 58. All were recruited as in Experiment 1. The apparatus was that described for Experiment 1.
Procedure. The subjects were exposed to the same instructions as those in Experiment 1 . They received three conditions. For the DRH condition, the subjects had to produce three responses within $3 \mathrm{sec}$ in order to produce an outcome. If the response requirement was not satisfied before the $3 \mathrm{sec}$ had elapsed, the timer started again and the subjects had to produce another three responses in $3 \mathrm{sec}$. The number of responses produced before these criteria were met for each successive DRH schedule was recorded, and these numbers formed the successive ratio requirements in the VR condition as described in Experiment 1 . In a third condition, the subjects responded on a VI schedule that was yoked to the temporal distribution of outcomes obtained in the DRH condition. The subjects all experienced the DRH condition prior to the other two conditions. All other aspects of the procedure were as in Experiment 1.

\section{Results and Discussion}

Table 2 presents the number of responses per minute, the number of outcomes obtained per minute, and the probability that a response would be followed by an outcome for the three conditions in Experiment 2. The subjects responded faster in the VI than in the VR condition, and faster in the VR than in the DRH condition. An ANOVA revealed a significant difference between the groups $[F(2,30)=6.70, p<.01]$. Subsequent Tukey's $H S D$ tests revealed that the VI and DRH conditions differed significantly $(p<.01)$. No other pairwise comparisons proved significant ( $p s>.05$ ). Greater numbers of outcomes were obtained per minute in the VR condition than in the DRH or VI conditions $[F(2,30)=6.83$, $p<.01]$. The probability that an outcome would follow a response was smaller in the VI than in the other two conditions; this probability was the same in the VR and DRH conditions. An ANOVA revealed a significant difference between the conditions $[F(2,30)=9.38$, $p<.01]$.

Figure 2 displays the mean rating of the effectiveness of a response in causing the outcome in each condition. Inspection of these data demonstrates that the responses in the VI and VR conditions were rated as equally causally effective, but both these conditions promoted higher ratings of the causal effectiveness of responses than did the DRH schedule condition $[F(2,30)=4.42, p<.05]$. Tukey's $H S D$ tests revealed that the VR and VI conditions did not differ $(p>.05)$, but that the DRH condition differed significantly from each of the other two conditions ( $p \mathrm{~s}<.05$ ). These results confirm the prediction that responses in the DRH condition would be rated as less causally effective than those in the VR condition, despite the probability that an outcome would follow a response

Table 2

Mean Responses per Minute, Outcomes per Minute, and Probability That an Outcome Would Follow a Response (O|R), for all Conditions in Experiment 2

\begin{tabular}{lrrr}
\hline & DRH & VR & VI \\
\hline Responses/minute & 38.75 & 56.75 & 70.81 \\
Outcomes/minute & 5.75 & 7.75 & 5.75 \\
Probability $(O \mid R)$ & 0.15 & 0.15 & 0.10 \\
\hline
\end{tabular}

Note $-\mathrm{DRH}=$ differential reinforcement of high rate; $\mathrm{VR}=$ variable ratio; $\mathrm{VI}=$ variable interval. 


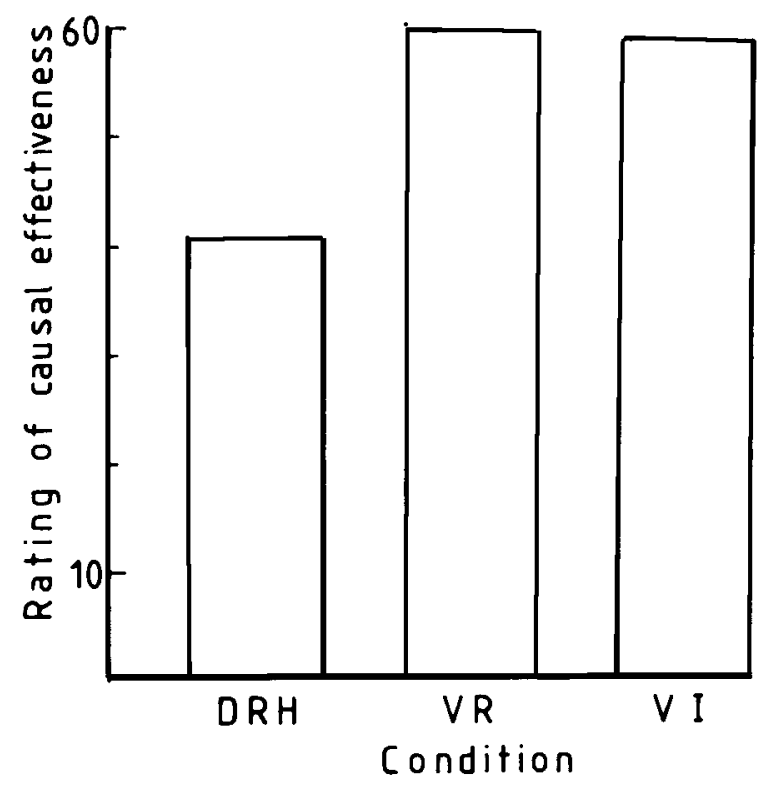

Figure 2. Results from Experiment 2: Mean ratings of the causal effectiveness of responses performed in each condition. DRH, differential reinforcement of high rate; VR, variable ratio.

being equal in the two conditions. This result was unlikely to be the sole effect of the greater temporal distribution of outcomes in the DRH compared with the VR schedule, since in a VI condition, in which the temporal distribution of outcomes was matched to that in the DRH condition, ratings of the causal effectiveness of responses were higher than in the DRH condition. These findings support the interpretation of the influence of the schedule of outcome presentation outlined above: responses produced on a schedule that requires temporal grouping of responses are rated as less causally effective than those produced on similar schedules that lack the temporalspacing requirement.

\section{GENERAL DISCUSSION}

A possible interpretation of the influence of schedules of outcome presentation on human judgment of causality was examined in the present experiments. This view, outlined by Reed (1991a), took as its starting point observations made about nonhuman performance on schedules of reinforcement. When exposed to an instrumental freeoperant schedule of reinforcement, animals typically respond with a variety of interresponse times (IRTs; cf. Morse, 1966). On schedules that arrange reinforcement for the first response following a certain period of time (e.g., VI schedules), it is more likely that reinforcement will follow a long IRT as opposed to a short IRT (Wearden $\&$ Clark, 1988). This relationship does not necessarily hold for schedules that lack a temporal requirement. For the procedure concerned with causality judgment in humans, Reed (1991a) likewise suggested that humans would respond with a variety of IRTs on the space bar of a com- puter. On a VI schedule (relative to a VR schedule), this made it more likely that responses produced in temporal isolation from other responses would be followed by an outcome. If such responses were assumed by the subject to be solely responsible for the outcome, the subject might rate these temporally isolated responses as more causally effective than responses produced on schedules that do not arrange outcomes following temporally isolated responses. On the latter schedules, a number of responses produced in close temporal proximity to the outcome might be viewed as jointly responsible for the outcome, and thus the rating of the causal effectiveness of a single response would be low.

The two experiments reported here are consistent with the account of human causality judgment that stresses the local context of responding prior to the occurrence of the outcome as a critical factor in human causal attribution. In Experiment 1, responses produced on a schedule that required temporally isolated responses preceding an outcome were rated as more causally effective than responses with a similar probability of producing an outcome but that were not necessarily temporally spaced. In contrast, when groups of responses were required to be temporally close to the outcome, the human rating of the causal efficacy of a response was low, relative to a condition that was matched in terms of the probability that an outcome would follow a response but that did not require that these responses be emitted in close temporal proximity to the outcome (Experiment 2).

The results from Experiment 1, however, suggest an alternative interpretation. Dickinson (1985) noted that the potential for control over the occurrence of outcomes may be an important factor in determining the level of causal efficacy attributed to a response in a particular situation. In Experiment 1, the potential for control over the $o c-$ currence of outcomes was greater in the DRL schedule than in the other two schedules employed. If the subject learned the appropriate relationship between responding and outcome presentation, there would be a high probability that a response would be followed by an outcome. Such a relationship may have developed over the course of training, so that although the overall probability of an outcome, given a response, was not markedly different from that in the other conditions, this measure was higher during the later stages of the DRL condition. If the later stages of exposure to a contingency are most important in determining ratings of causal effectiveness, responses produced during exposure to the DRL condition would be rated as more effective than responses produced during exposure to the other conditions, in which there was much less opportunity for learning such a relationship. Although attractive, such an interpretation would need to account for the fact that ratings of responses produced during exposure to the DRH condition in Experiment 2 were low relative to the other conditions in that experiment. There was as much scope for control over the occurrence of outcomes in the DRH schedule as in the DRL schedule (albeit outcomes necessitated more responses in 
the DRH than in the DRL condition), and so ratings of causal efficacy should also have been high in the DRH condition. The fact that ratings of responses produced during the DRH condition were low causes problems for this interpretation of the data.

It was also evident that the temporal distribution of outcomes, irrespective of the temporal distribution of responses, was not in itself responsible for the high rating of causal effectiveness of responses. A VI condition matched to the DRL schedule in terms of the temporal distribution of outcomes produced relatively low ratings of causal effectiveness (Experiment 1). In contrast, the VI schedule in Experiment 2 that was matched to the DRH schedule in terms of the temporal distribution of outcomes produced a relatively high rating of the causal effectiveness of the responses produced during exposure to it. This indicated that the temporal distribution of outcomes is not the sole determinant of human judgment of causality. This possibility was also directly examined, and ruled out, by Reed (1992; Experiment 3).

Similarly, it is difficult to understand how variations in the rate of outcome presentation could have been solely responsible for the present results. Although this factor can influence the rating of causal effectiveness given to responses under certain conditions (see Alloy \& Abramson, 1979; Wasserman, 1990), no clear relationship between the rates of outcome presentation emerged in the present studies, either within or between experiments. Any account of causal attribution based purely on rates of outcome presentation would need to establish the fact that whenever rates of outcome presentation were high (or low), the ratings of causal effectiveness of responses were high (or low). On the basis of previous research, it is likely that rates of outcome presentation would be positively related to judgments of causal effectiveness: Alloy and Abramson (1979) established that the greater the frequency of outcomes, the greater the rating of causal effectiveness. In fact, the highest rating of causal attribution was given to the condition with the greatest rate of outcome presentation-that is, the VR condition in Experiment 2. However, this is about as far as clear parallels in the two scores go. For example, rates of outcome presentation were lowest in the DRL condition in Experiment 1, but ratings of the causal effectiveness of responses made during exposure to this condition were relatively high. Specifically, ratings of the causal effectiveness of the responses produced during exposure to the DRL schedule in Experiment 1 were higher than those given to responses produced during exposure to the DRH schedule in Experiment 2, which had a higher rate of outcome presentation. It may well be that rate of outcome presentation does influence judgments of causal effectiveness, but the view that this in itself provides a complete explanation is not well supported by the present data.

It should be mentioned that in each of the present experiments, the condition of principal theoretical interest (i.e., the DRL schedule in Experiment 1 and the DRH schedule in Experiment 2) was presented to the subjects first. This was necessary for comparisons between this schedule and appropriately controlled VR and VI conditions to be made. It is possible that this procedure may be resulted in the judgments of causal effectiveness being subject to some order effect. Although Shanks (1985) found no evidence that order of presentation of conditions in a similar set of experiments influenced judgments, this issue warrants further discussion. The possibility that order effects occurred in the present studies is rendered unlikely by the fact that, in Experiment 1, the DRL condition was rated more causally effective than the subsequent conditions, and in Experiment 2, the DRH condition was rated less causally effective than the subsequent conditions. If order effects were invoked to explain these results, the reasons why they should influence subjects consistently in one manner in one experiment and consistently in the opposite manner in the second experiment would need to be specified. In fact, previous studies with VR and VI schedules have shown the same pattern of results between these conditions (i.e., other things being equal, the VI schedule promotes higher ratings of causal effectiveness of responses than does the VR schedule), irrespective of the order of presentation. Thus, although it is acknowledged that order effects could, in principle, play a part in the present results, this possibility is rendered less likely by an examination of the pattern of results in the two present experiments.

A final observation on the present results is worth making, with respect to the relationship between the schedule and the rates of responses made during exposure to the contingency. In nonhuman conditioning on free-operant schedules of reinforcement, VR schedules produce a much higher response rate than VI schedules do (see Ferster \& Skinner, 1957). In the present experiments, the converse relationship between schedule and response rate held: VI schedules produced a higher rate. Two points need to be mentioned here. First, the present experiment was designed to assess the effect of a schedule of outcome presentation on human judgments of causality. It is not necessarily the case that an outcome is a reinforcer, and neutral outcomes may not have the same effects on response rates as do outcomes that could be classified as rewards. Second, in some studies, in which the outcomes have been manipulated so that each outcome was worth a number of points (e.g., Shanks \& Dickinson, 1991), it has been found that response rates in experiments dealing with human causality judgments do appear to follow a pattern similar to those observed in nonhuman conditioning with free-operant schedules of reinforcement.

In sum, these results provide support for both the local context view of the factors influencing human causal attribution and the functional equivalence between the factors that influence conditioning and those that influence human causal attribution. In this case, the factors impli- 
cated in instrumental performance on free-operant schedules of reinforcement were found to exert an influence on human judgments of causal efficacy.

\section{REFERENCES}

Alloy, L. B., Abramson, L. Y. (1979). Judgment of contingency in depressed and non-depressed students: Sadder but wiser? Journal of Experimental Psychology: General, 108, 441-485.

Chapman, G. B., Robrins, S. J. (1990). Cue interaction in human contingency judgment. Memory \& Cognition, 18, 537-545.

Dickinson, A. (1985). Actions and habits: The development of be havioural autonomy. Philosophical Transactions of the Royal Society: Series $B, 308,67-78$.

FERSTER, C. B., \& SKINNER, B. F. (1957). Schedules of reinforcement. New York: Appleton-Century-Crofts.

Morse, W. H. (1966). Intermittent reinforcement. In W. K. Honig (Ed.), Operant behavior: Areas of research and application (pp. 52108). New York: Appleton-Cenury-Crofts.

Neunaber, D. J., Wasserman, E. A. (1986). The effects of unidirectional versus bidirectional rating procedures on college students' judgments of response-outcome contingency. Learning \& Motivation, 17, 162-179.

Peele, D. B., Casey, J., \& Silberberg, A. (1984). Primacy of interresponse-time reinforcement in accounting for rates under variableratio and variable-interval schedules. Joumal of Experimental Psychology: Animal Behavior Processes, 10, 149-167.

REED, P. (1989). Marking effects in instrumental performance on DRH schedules. Quarterly Joumal of Experimental Psychology, 41B, 337-353.

REED, P. (1992). Effect of the schedule of outcome presentation on human judgments of causality. Manuscript submitted for publication.

ShAKLEE, H., TuCKer, D. (1980). A rule analysis of judgments of covariation between events. Memory \& Cognition, 8, 459-467
Shanks, D. R. (1985). Forward and backward blocking in human causality judgment. Quarierly Joumal of Experimental Psychology, 37B, 1-21.

Shanks, D. R., Dickinson, A. (1987). Associative accounts of causality judgment. In G. H. Bower (Ed.), The psychology of learning and motivation (Vol. 21, pp. 229-261). London: Academic Press.

Shanks, D. R., Dickinson, A. (1991). Instrumental judgment and performance under variations in action-outcome contingency and contiguity. Memory \& Cognition, 19, 353-360.

Shanks, D. R., Pearson, S. M., Dickinson, A. (1989). Temporal contiguity and the judgment of causality by human subjects. Quarterly Joumal of Experimental Psychology, 41B, 139-159.

WARD, W. C., JENkINS, H. M. (1965). The display of information and the judgment of contingency. Canadian Journal of Psychology, 19, 231-241.

Wasserman, E. A. (1990). Detecting response-outcome relations: Toward an understanding of the causal texture of the environment. In G. H. Bower (Ed.), The psychology of leaming and motivation (Vol. 26, pp. 27-82). London: Academic Press.

Wasserman, E. A., Chat losh, D. L., Neunaber, D. J. (1983). Perception of causal relations in humans: Factors affecting judgments of response-outcome contingencies under free-operant procedures. Learming \& Motivation, 14, 406-432.

WeArDEN, J. H., Clark, R. B. (1988). Interresponse time reinforcement and behavior under aperiodic reinforcement schedules: $A$ case study using computer modelling. Journal of Experimental Psychology: Animal Behavior Processes, 14, 200-211.

ZEILER, M. D. (1977). Schedules of reinforcement: The controlling variables. In W. K. Honig \& J. E. R. Staddon (Eds.), Handbook of operant behavior. Englewood Cliffs, NJ: Prentice-Hall.

(Manuscript received May 8, 1991; revision accepted for publication December 24, 1991.) 